\title{
L'ADAPTATION DES CENTRALES NUCLÉAIRES AUX BESOINS DU RÉSEAU ÉLECTRIOUE
}

\section{PROGRAMME DE LA CONFÉRENCE}

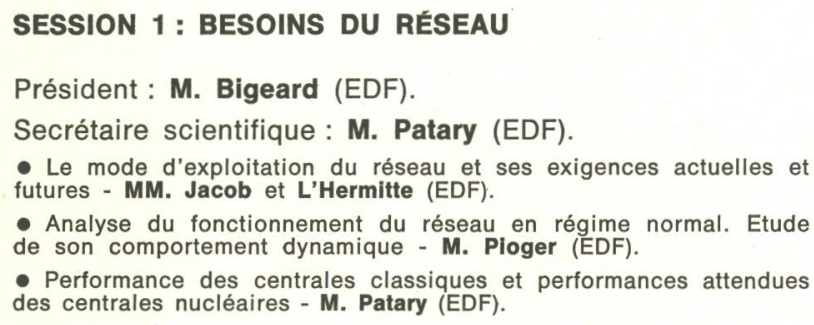

- Analyse du fonctionnement du réseau en régime normal. Etude de son comportement dynamique - M. Ploger (EDF).

- Performance des centrales classiques et performances attendues des centrales nucléaires - M. Patary (EDF).

\section{SESSION 2 : PERFORMANCES DES RÉACTEURS A EAU}

Président : M. Panossian (Framatome).

Secrétaire scientifique: $\mathbf{M}$. Guesdon (Framatome).

- Performance des chaudières PWR. Dispositif de manœuvrabilité accrue - M. Gautier (Framatome) et M. Coqueriaux (EDF).

- Comportement des chaudières PWR vis-à-vis des incidents du réseau - M. Bruyère (Framatome).

- Essais de téléréglage à Fessenheim - M. Coqueriaux (EDF) et

M. Bruyère (Framatome)

- Essais à Tricastin 3 du dispositif de manœuvrabilité accrue

M. Sengler (Framatome) et M. Queri (EDF).

\section{SESSION 3 : IMPACT DU SUIVI DU RÉSEAU}

\section{SUR LES COMPOSANTS}

Président : M. Delayre (Technicatome).

Secrétaire scientifique: M. Mabile (Technicatome).

- Sollicitations imposées aux combustibles par les variations de puissance. Problèmes posés et essais de qualification - M. Traccucci (Fragéma) et M. Marin (CEA).

- Essais de combustible dans la CAP - M. Bergeron (CEA) et M. Mabile (Technicatome).

- Conséquences du suivi du réseau pour les composants mécaniques de la chaudière - MM. Assedo et Dubreux (Framatome).

- Conséquences du suivi du réseau sur les équipements de contrôle-commande des grappes d'arrêt et de régulation - MM. Pilavoine et Bellegro (Framatome).

\section{SESSION 4 : PROBLĖMES D'APPLICATION \\ ET CONCLUSIONS}

Président: $\mathbf{M}$. Bacher (EDF).

Secrétaire scientifique: M. Hourtoulle (EDF).

- Aide au pilotage ; effluents - M. Coqueriaux (EDF) et M. Darraud (Framatome).

- Caractéristiques particulières des réacteurs rapides (aspects fonctionnels, aspects technologiques) - MM. Cazalet, Marbach (CEA), M. Debru (Novatome) et M. Decuyper (NERSA).

- Mise en œuvre sur les PWR - M. Baudry (EDF).

- Conclusion - M. Bacher (EDF). $\sum a$ a Société Française d'Energie Nucléaire et la Société des Electriciens, des Electroniciens et des Radioélectriciens ont organisé les 8 et 9 décembre 1981, à Paris, une conférence nationale sur " l'adaptation des centrales nucléaires aux besoins du réseau électrique ".

On sait qu'un des objectifs du producteur d'électricité - EDF — est d'être toujours en mesure d'adapter la production d'électricité à la consommation. La contribution grandissante des centrales nucléaires à la production d'électricité implique de plus en plus que celles-ci participent au suivi de la consommation et à l'adaptation aux variations de production.

L'objet de la conférence de la SFEN et de la SEE était de cerner les différents problèmes posés par l'introduction de cette plus grande souplesse dans le fonctionnement quotidien des tranches nucléaires.

Les quatre secrétaires scientifiques de la conférence, MM. Guesdon, Hourtoulle, Mabile et Patary, ont bien voulu établir la synthèse de l'ensemble des travaux. Nous tenons à les remercier vivement pour leur très aimable collaboration.

Nous publions par ailleurs deux communications concernant un aspect particulièrement important de la question: l'impact du suivi $d u$ réseau sur les composants du réacteur nucléaire. Elles sont dues à MM. Marin et Traccucci, ainsi qu'à MM. Assedo et Dubreux.

Qu'ils en soient remerciés, de même que tous les conférenciers qui nous ont permis d'établir ce dossier de synthèse. 